\title{
Liars Dominationset on fuzzy Graphs under Join, Corona, and Lexicographic Products
}

\author{
S. Roseline Mary' S.Ruban Raj
}

\begin{abstract}
A set $L \subseteq V(G)$ of a fuzzy graph $G=(V, E)$ is a liar's dominating set if (1) for all $v \in V(G),|N[v] \cap L| \geq 2$ and (2) for each pair $(u, v) \in V(G)$ of unmistakable vertices, $\mid N[u] \cup \quad N[v]$ $\cap L \mid \geq 3$. In this paper, we consider the liar's control number of some center graphs. Crown result of twofuzzy graphs which is undifferentiated from the idea crown item activity in fresh graph hypothesis is characterized. The level of an edge in crown result of fuzzy graphs is acquired. Additionally, the level of an edge in fuzzy graph framed by this activity as far as the level of edges in the given fuzzy graphs in some specific cases is found. In addition, it is demonstrated that crown result of two fuzzy graphs is compelling when two fuzzy graphs are powerful fuzzy graphs.

Keywords : Corona product, degree, lexicographic, fuzzy graph
\end{abstract}

\section{INTRODUCTION}

Horary clarified an interesting application with regards to casting a ballot circumstances utilizing the idea of control. The investigation of ruling sets in fuzzy graphs was started by Ore and Berge, the control number, autonomous mastery number are presented by Hedetniemi. As well as, Rosenfeld presented the thought of fuzzy graph and a few fuzzy analogs of graphs theoretic ideas, for example, paths, cycles and connectedness. S.Somasundram talked about mastery in fuzzy graph.They characterized control utilizing powerful edges in fuzzygraph. Chandrasekaran talked about control in fuzzy graph utilizing solid curves. We additionally talk about control, autonomous mastery and ideal control in fuzzy graph utilizing solid circular segments. Nagoorgani talked about fuzzy autonomous overwhelming sets. In this paper, the discussion about when the fuzzy graph has flawless mastery and free control number. The significant definitions are being provided and do clarification about the models. Some fuzzygraphs likewise contrasted and the fresh case.

Revised Manuscript Received on July 22, 2019

S. Roseline Mary, S.Ruban Raj

Department of Mathematics, Loyola College, Tiruvannamalai

Department of Mathematics, RVS College of Arts and Science, Tiruchirappalli

\section{Definitions:}

Suppose $G=(\sigma, \mu)$ be a fuzzygraph. A subset $L$ of $V$ is saidto be a dominating set of Gonly if for each $v \in V(G), V$ - L there is an existenceof $\mathrm{L}$ such that $\mathrm{u}$ do domination of $\mathrm{v}$.

Definition :A dominating set $\mathrm{L}$ of a fuzzy graph $\mathrm{G}$ is named as the minimal dominating set of $\mathrm{G}$ if there do not have existence any dominating set of $\mathrm{G}$ which have cardinality is minimum than the cardinality of L. Least cardinality among all base dominating set in $\mathrm{G}$ is called mastery number of $\mathrm{G}$ is signified by $\gamma(\mathrm{G})$.

Significant hypothesis for liar mastery.

Hypothesis 1. In the event that $L \subseteq V(G)$ is a liar's dominating set then every part of $(\mathrm{L})$ contains in any event three vertices.

Hypothesis 2. For each associated fuzzy graphG of request $\mathrm{s}$ $\geq 3$ we have $\gamma \mathrm{y} 2(\mathrm{G}) \leq \gamma \mathrm{LR}(\mathrm{G})$, and, in the event that $\mathrm{G}$ has least degree $\delta(\mathrm{G}) \geq 2$, at that point $\gamma \mathrm{y} 2(\mathrm{G}) \leq \gamma \mathrm{LR}(\mathrm{G}) \leq$ $\gamma \mathrm{y} 3(\mathrm{G})$. The following hypothesis gives essential and adequate conditions for $\mathrm{D} \subseteq \mathrm{V}(\mathrm{G})$ to be a liar's dominating set.

Hypothesis 3. A dominating set $L \subseteq V(G)$ is a liar's overwhelming set if and just if Ldual rules each $n \in V(G)$ and $|\mathrm{N}[\mathrm{u}] \cup \mathrm{N}[\mathrm{v}] \cap \mathrm{L}| \geq 3$ for each pair $\mathrm{u}, \mathrm{v}$ of unmistakable vertices.

In this paper, we consider the liar's mastery number for center graphs of some notable diagrams The complete diagram $T(G)$ of a graphG is a chart with the end goal that the vertex set of $U$ compares to the vertices and edges of $G$ and two vertices are nearby in $\mathrm{U}$ if their relating components are either contiguous or episode in G. All out graphs are speculations of line charts. The line diagram $Q(G)$ of a straightforward graphG is acquired by partner a vertex with each edge of the chart and associating two vertices with an edge if the comparing edges of $G$ share a vertex for all intents and purpose. The center graph $\mathrm{M}(\mathrm{G})$ is the graph acquired from $\mathrm{G}$ by embedding another vertex into each edge of $\mathrm{G}$ and after that joining these new vertices by edges, which lie on the

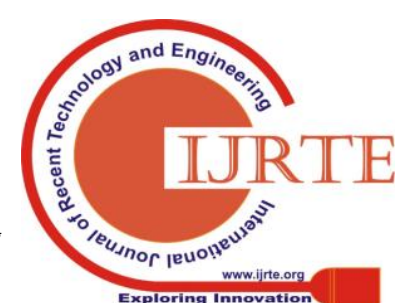


adjoining edges of G. Specifically, we consider the center graphs in light of the fact that these graphs are between all out graphs and line diagrams. In actuality, issues, each edge compares cost $[1,2]$
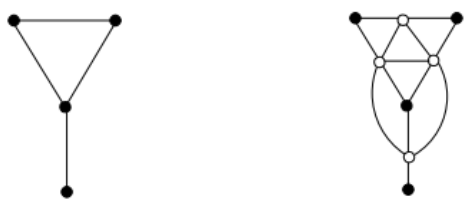

\section{Liar's domination in the corona of fuzzy graphs}

Liar Domination in the Corona of fuzzy Graphs is defined as follows. The crown of two fuzzy graphs $\mathrm{A}$ and $\mathrm{B}$, meant by $\mathrm{A}$ $\circ \mathrm{B}$, is the fuzzy graph gotten from A by taking a duplicate $\mathrm{Hv}$ of Band framing the join hmi $+\mathrm{Hv}=\mathrm{m}+\mathrm{Hv}$

Hypothesis: Let A be a nontrivial associated fuzzy graph and $\mathrm{B}$ be any diagram. At that point $\mathrm{V}(\mathrm{A})=\operatorname{Int}(\mathrm{A} \circ \mathrm{B})$. Verification. Guarantee 1. Int $(A \circ B) \subset V(A)$ Let $n \in \operatorname{Int}(A \circ$ $B)$. Assume $n / \in V(A)$. At that point $n \in V(A \circ B) \backslash V(A)$. At that point $n \in V(\mathrm{Hv})$ for some $m \in V(A)$. Since $m \in N A \circ B$ $(n)$ and $m z \in E(A \circ B)$ for each $z \in N A \circ B(n) \backslash\{m\}, n$ isn't an inside vertex of $A \circ B$. It pursues that $n \in V(A)$.

Proof : $V(A) \subset \operatorname{Int}(A \circ B)$ Let $m \in V(A n)$ and $e \in N A \circ B(m)$. Assume $u \in V(A)$. At that point there exists $s \in V(H v)$ with the end goal that $\mathrm{d} A \circ \mathrm{B}(\mathrm{z}, \mathrm{e})=2$. In the event that $\mathrm{e} / \in \mathrm{V}(\mathrm{An})$, at that point $e \in V(\mathrm{Hv})$. Since $A$ is non-paltry and associated, there exists $\mathrm{j} \in \mathrm{V}(\mathrm{A}) \cap \mathrm{NA}(\mathrm{m})$. Along these lines, $\mathrm{dA} \circ \mathrm{B}(\mathrm{e}$, $\mathrm{j})=2$. Subsequently, $m \in \operatorname{Int}(A \circ B)$. Along these lines $V(A)$ $=\operatorname{Int}(\mathrm{A} \circ \mathrm{B}) \cdot[2,6]$

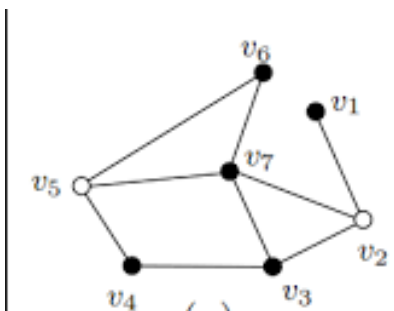

(a)

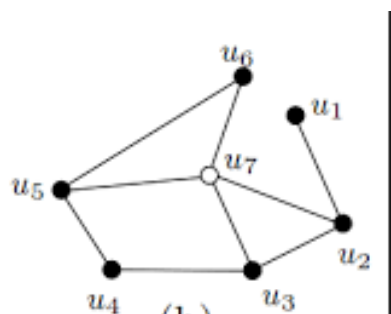

(b)

\section{Liar's domination in the join of fuzzy graphs}

Theorem: The join $A+B$ of two fuzzy graphs $A$ and $B$ is the fuzzy graph with vertex set $\mathrm{V},(\mathrm{A}+\mathrm{B})=\mathrm{V}(\mathrm{A}) \cup \mathrm{V}(\mathrm{B})$ and edge set $E(A+B)=E(A) \cup E(B) \cup\{x y: x \in V(A), v \in V$ (B) $\}$.

Let $A$ and $B$ be associated fuzzy graphs. On the off chance that $\gamma \operatorname{Id}(\mathrm{A})=1$ and $\gamma \mathrm{Id}(\mathrm{B})=1$, at that point $\mathrm{A}+\mathrm{B}$ has no inside dominating set.

Proof: Let $\mathrm{M} 1=\{\mathrm{y}\}$ and $\mathrm{M} 2=\{\mathrm{x}\}$ be the $\gamma$ Id-sets of $\mathrm{A}$ and $\mathrm{B}$, separately. Assume $\mathrm{A}+\mathrm{B}$ has an inside ruling set $\mathrm{D}$ and $\mathrm{D}$ $\subset \mathrm{V}(\mathrm{A})$. Let $\mathrm{m} \in \mathrm{D}$. Since $\mathrm{x} \in \mathrm{NA}+\mathrm{B}(\mathrm{m})$, there exists $\mathrm{n} \in$ $\mathrm{NA}+\mathrm{B}(\mathrm{m})$ with the end goal that $\mathrm{dA}+\mathrm{B}(\mathrm{x}, \mathrm{n})=2$. On the off chance that $n \in V(B)$, at that point $n / \in N B(x)$ which is a logical inconsistency since $\mathrm{M} 2$ is a ruling arrangement of $\mathrm{B}$. Then again, in the event that $\mathrm{v} \in \mathrm{V}(\mathrm{An})$, at that point $\mathrm{v} / \in$ $\mathrm{NA}+\mathrm{B}(\mathrm{x})$ which is again a logical inconsistency to the meaning of $\mathrm{A}+\mathrm{B}$. Thus, if $\mathrm{D} \subset \mathrm{V}(\mathrm{B})$ or $\mathrm{D}=\mathrm{DA} \cup \mathrm{DB}$ where $\mathrm{DA} \subseteq \mathrm{V}(\mathrm{An})$ and $\mathrm{DB} \subseteq \mathrm{V}(\mathrm{B})$, at that point we achieve an inconsistency. Accordingly, $\mathrm{A}+\mathrm{B}$ do not possess an inside dominating set.

\section{Converse of the theorem:}

The opposite of Theorem isn't valid. To see this, consider the fuzzy graphs A and A + B in underneath figure. Let B = J2. At that point it very well may be checked that the inside dominating arrangement of $\mathrm{A}+\mathrm{B}$ does not exists. Besides, Int $(\mathrm{A})=\emptyset$ and Int $(\mathrm{J} 2)=\emptyset$
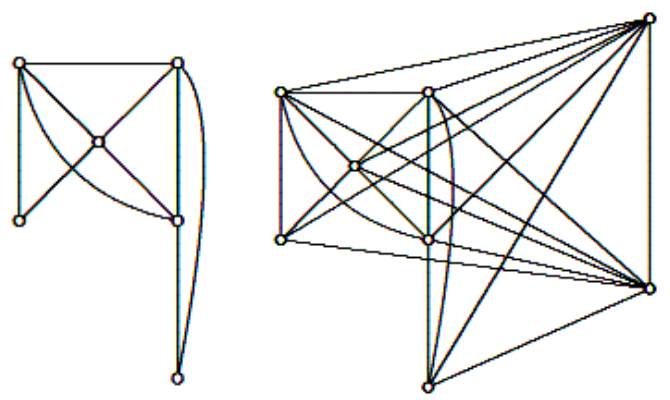

Figure: liar domination jointof these two do not exist

\section{Liar's domination in the lexicographic product of fuzzy graphs}

- In a common realistic hypothetical learning, the different sorts of lexicographic items or huge structure A - B of the two kinds of diagrams $A n$ and $B$ is an indistinguishable fuzzy graph for instance :

- the vertex set of $\mathrm{A} \cdot \mathrm{B}$ is the main Cartesian product by the $\mathrm{V}(\mathrm{A}) \times \mathrm{V}(\mathrm{B})$; and

- Two variant vertices $(\mathrm{x}, \mathrm{y})$ and $(\mathrm{m}, \mathrm{n})$ are normally adjacent in $\mathrm{A} \cdot \mathrm{B}$ if and only if either $\mathrm{x}$ is adjacent to $\mathrm{m}$ in $\mathrm{A}$ or $\mathrm{x}=\mathrm{m}$ and $\mathrm{y}$ is also adjacent to $\mathrm{y}$ in $\mathrm{B}$.

On the off chance that the edge relations of the two diagrams are organization relations, at that point the edge connection of their lexicographic item is the comparing lexicographic request.

The lexicographic item was first concentrated by Felix Hausdorff. As appeared, the issue of perceiving whether a

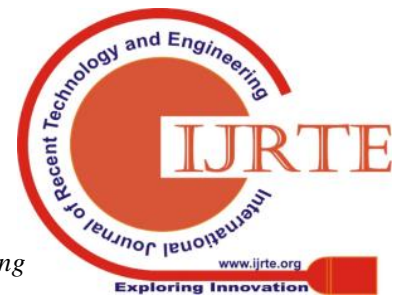


chart is a lexicographic item is equal in unpredictability to the diagram isomorphism issue.

Non -commutative nature is being displayed byit: $\mathrm{A} \cdot \mathrm{B} \neq \mathrm{B} \cdot \mathrm{A}$. However , a distributive law is being satisfied w.r.t the non-similar type of disjoint union: $(\mathrm{X}+\mathrm{Y}) \cdot \mathrm{Z}=\mathrm{X} \cdot \mathrm{Z}+\mathrm{Y} \cdot \mathrm{Z}$. additionally, satisfaction of an identity w.r.t the complementation law: $C(A \cdot B)=C(A)$ - C(B).[3,7]

The numerous form of a lexicographic product might be easily deliberated from below mentioned factors:

$\alpha(\mathrm{A} \cdot \mathrm{B})=\alpha(\mathrm{A}) \alpha(\mathrm{B})$.

The clique count of a lexicographic product is having the multiplicative nature:

$\omega(\mathrm{A} \cdot \mathrm{B})=\omega(\mathrm{A}) \omega(\mathrm{B})$.

The chromatic number of a lexicographic item is equivalent to the b-overlay chromatic number of $\mathrm{A}$, for $\mathrm{b}$ equivalent to the chromatic number of $\mathrm{B}$ :

$\chi(\mathrm{A} \cdot \mathrm{B})=\chi_{\mathrm{b}}(\mathrm{A})$, where $\mathrm{b}=\chi(\mathrm{B})$.

The lexicographic result of two fuzzy graphs is an ideal fuzzy graph if and just if the two elements are flawless

Theorem: Suppose A and B are non- similar fuzzy graphs of connected types. Proof that:Int $(\mathrm{A}[\mathrm{B}])=\operatorname{Int}(\mathrm{A}) \times \operatorname{Int}(\mathrm{B})$.

Proof. Suppose (m, p) $\in \operatorname{Int}(A[B]), n \in N A(x)$ and $q \in N B(p)$. because $(n, q) \in N A[B]((m, p))$, here are present $(o, r) \in$ $\mathrm{NA}[\mathrm{B}]((\mathrm{m}, \mathrm{p}))$ for instance, $\mathrm{dA}[\mathrm{B}]((\mathrm{o}, \mathrm{r}),(\mathrm{n}, \mathrm{q})=2$. thus, $\mathrm{o} \in$ $\mathrm{NA}(\mathrm{m}), \mathrm{dA}(\mathrm{n}, \mathrm{o})=2$, and chose $\mathrm{r} \in \mathrm{NB}(\mathrm{p})$. so, $\mathrm{m} \in \operatorname{Int}(\mathrm{A})$ and $\mathrm{p} \in \operatorname{Int}(\mathrm{B})$. it indicates that $(\mathrm{m}, \mathrm{p}) \in \operatorname{Int}(\mathrm{A}) \times \operatorname{Int}(\mathrm{B})$.

$\operatorname{Suppose}(m, p) \in \operatorname{Int}(A) \times \operatorname{Int}(B)$ and $(n, q) \in N A[B]((m, p))$. Thus, $n \in N A(m)$ or $m=n$ and $q \in N B(p)$. Suppose $n \in N A(m)$. Hence $m \in I n t(A)$, existence of $o \in N A(m)$ for exampledA(n, $o)=2$. So, $(o, p) \in N A[B]((m, p))$, here $d A[B]((o, p),(n, q)=$ 2. moreso, presence of $r \in N B(p)$ for example $d B(q, r)=2$ hence $p \in \operatorname{Int}(B)$. It do following of $(m, p) \in \operatorname{Int}(A[B])$.

Thus proof, $\operatorname{Int}(\mathrm{A}[\mathrm{B}])=\operatorname{Int}(\mathrm{A}) \times \operatorname{Int}(\mathrm{B})$.

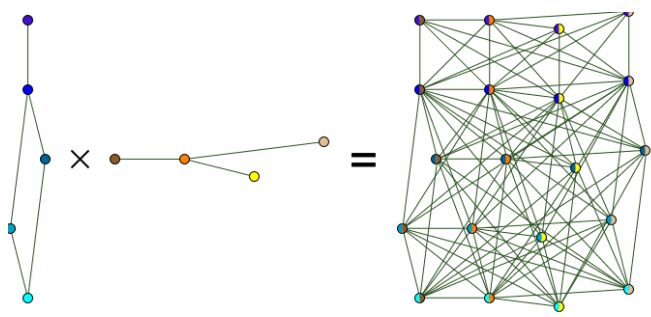

Figure: lexicographic product

\section{CONCLUSION}

Underjoin,lexographic and other operations ,the liar dominate setwith theorms and proof are being shown on the basis of fuzzy graphs. The particular conditions are also being displayed with the help of it.

\section{REFERENCES}

1. A.Nagoorgani and M.BasheerAhamed, Order and size in fuzzy graph, Bulletin of Pure and Applied Sciences, 22E (1) (2003) 145-148

2. K.Radha and N.Kumaravel, The degree of an edge in Cartesian product and composition of two fuzzy graphs, Intern. J. Applied Mathematics \& Statistical Sciences, 2(2) (2013) 65-78.

3. K.Radha and N.Kumaravel, The degree of an edge in union and join of two fuzzy graphs, International Journal of Fuzzy Mathematical Archive, 4(1) (2014) 8-19.

4. A.Roseneld, fuzzy graphs, in: L.A.Zadeh, K.S. Fu. K.Tanaka, M.Shimura, Fuzzy sets and Their Applications to Cognitive and Decision Processes, Academic Press, New York, (1975) 77-95.

5. R.Frucht and F.Harary, on the corona two graphs, Aequationes Math., 4 (1970) 322- 325

6. F.Hdrary, Graph Theory, Addition-Wesley pub. Co., London, 1969.

7. J.N.Mordeson and C.S.Peng, Operation on fuzzy graphs, Information Sciences, 79 (1994) 159-170.

8. Nagoorgani, A., and Vadivel, P., Fuzzy independent dominating set, Adv. in Fuzzy sets and system 2(1) (2007), 99-108.

9. Nagoorgani,A., Vadivel,P., Relations between the parameters of Independent Domination and Irredundancein Fuzzy Graph, International Journal of Algorithms, Computing and Mathematics, Volume 2, Number 1, pp. 15-19, 2009.

10. Rosenfeld, A., Fuzzy graphs in: Zadeh, L.A., Fu, K.S., Shimura, M (eds),Fuzzy Sets and Their Applications, Academic Press, New York, 1975. 